\title{
Aproximación al diseño de un instrumento sobre actitudes y creencias hacia la investigación en estudiantes universitarios
}

\author{
A study on the design of an instrument regarding University students attitudes and beliefs towards \\ research \\ Jamely Abigail Gómez-López a , Javier Moreno-Tapia ${ }^{c}$, Coralia Juana Pérez-Maya ${ }^{c}$
}

\begin{abstract}
:
This report describes the progress in the design of a measurement instrument to assess the beliefs and attitudes of university students towards their training for the achievement of educational research competence. The authors use learning constructivist theories which consider an essential element to have a positive attitude towards learning while false beliefs do not position the learner in an optimum condition to reach the next step of development in a didactic situation. It is a quantitative study. It was applied to an intentional sample of undergraduate students in Educational Sciences, from the Autonomous University of the State of Hidalgo. The results show a reliability of .77. Thus, an analysis of the wording and appropriateness is made, as well as its correlation. Finally, a second version is proposed seeking to spread the application of the questionnaire for its validation.
\end{abstract}

Keywords:

Beliefs, Attitudes, Competence, Research

\section{Resumen:}

Este reporte describe el avance en el diseño de un instrumento de medición para valorar las creencias y actitudes de estudiantes universitarios hacia su formación para el logro de la competencia de investigación educativa. Pertenece al Proyecto: "Enseñanza y evaluación de la competencia de investigación". El sustento se basa en teorías constructivistas del aprendizaje, donde se considera un elemento esencial contar con una actitud positiva para aprender. Además de que las creencias falsas no posicionan al aprendiz en un estado óptimo para alcanzar el nivel de desarrollo próximo ante una situación didáctica. El estudio es de corte cuantitativo; se aplicó a una muestra intencional de estudiantes de la licenciatura en Ciencias de la Educación, de la Universidad Autónoma del Estado de Hidalgo. Los resultados muestran una fiabilidad de .77, por lo que se realiza un análisis de la redacción, pertinencia, así como su correlación. Por último, se propone una segunda versión, buscando difundir la aplicación del cuestionario para su validación.

\section{Palabras Clave.}

Creencias, Actitudes, competencia, investigación

Recibido: 13 de junio de 2018

Dictaminado: 29 de julio de 2018

Segunda versión: 5 de agosto de 2018

Aceptado: 28 de septiembre de 2018

\footnotetext{
a Egresada Especialidad en Docencia. Universidad Autónoma del Estado de Hidalgo. País: México, ORCID https://orcid.org/0000-0003-27758789 Email: jamelyuaeh@ hotmail.com

b Profesor Investigador en el área de Ciencias de la Educación, Universidad Autónoma del Estado de Hidalgo. País: México, ORCID https://orcid.org/0000-0003-4029-5440 Email: javier_moreno@uaeh.edu.mx

c Profesora Investigadora en el área de Ciencias de la Educación, Universidad Autónoma del Estado de Hidalgo. País: México, ORCID https://orcid.org/0000-0002-0850-3492 Email: cpm258@yahoo.com.mx
} 


\section{Introducción}

La formación para la investigación es una tarea importante para las universidades, como una competencia esencial de sus egresados. La Universidad Autónoma del Estado de Hidalgo (UAEH), a través de su oferta educativa con la Licenciatura en Ciencias de la Educación, busca desarrollar en sus alumnos, entre otras, la competencia de investigación e intervención educativa; sin embargo, a partir de algunos antecedentes se puede dar cuenta que existe poca producción de tesis en cada generación de egresados. Esto se podría deber a diversos aspectos y uno de los más importantes se encuentra en la normativa del reglamento escolar de la UAEH (2013), ya que en su capítulo II, referente a la titulación, específicamente en el artículo 85, menciona que para obtener el título de licenciatura se puede optar por la aplicación del examen CENEVAL.

En este sentido, Moreno (2011) señala que una meta dentro de las universidades es consolidar la investigación como eje central para la transformación de la educación superior. Sin embargo, a partir de esta consolidación se cruzan tanto elementos de nivel macro como son las políticas nacionales, las condiciones de financiamiento, las prioridades nacionales y regionales, así como elementos de nivel micro, como las políticas institucionales, cualificación de los académicos, infraestructura de apoyo, programas de formación. Es decir, todos estos elementos que se encuentran desde el punto de vista macro y micro influyen para impulsar la formación en investigación en las universidades.

Además, Estrada (2014) menciona que las universidades han encaminado algunas de sus acciones, al desarrollo de la competencia en investigación, porque esta competencia permite a los egresados universitarios integrarse a espacios que den solución a problemas profesionales desde una concepción científica e investigativa. Su formación y desarrollo se encuentra normalmente presente en los programas educativos de carreras universitarias, debido a que responde a intereses, necesidades y demandas de la sociedad y los recursos personales que posee el individuo.

No obstante, dentro del programa educativo de la Licenciatura en Ciencias de la Educación, hay asignaturas referentes a la promoción de la competencia de investigación y se espera desarrollar aprendizajes en este sentido. Por consecuencia, elaborar una investigación para el desarrollo de una tesis o la publicación de un artículo podría ser una meta que los estudiantes deberían de considerar, como mecanismo de titulación, debido a que curricularmente es muy valioso contar con la experiencia de haber realizado una investigación, como producto de la titulación (una tesis de pregrado). Esto facilita el proceso al momento de solicitar adscripción a un posgrado o cuando se hace una solicitud de empleo, ya que con ello se demuestra, a través de un producto académico, el dominio de habilidades para el trabajo independiente, sistemático y revela competencias en argumentación, comprensión lectora, escritura científica y en la mayoría de los casos, para el análisis de datos.
Sin embargo, Aldana (2012, p.375) menciona que es difícil encontrar en estudiantes de pregrado y a veces de posgrado, habilidades para la investigación; por tal motivo, indica que es necesario fomentar en los estudiantes habilidades básicas como:

- La lectura comprensiva y crítica que beneficia a la construcción del problema y textos que fundamenten las propuestas de investigación.

- La escritura lógica y coherente para desarrollar capacidades para transmitir las ideas claras a los lectores.

- El manejo de herramientas digitales, ya que ayudan a optimizar los tiempos y a trabajar de manera más rápida, además de convertirse en instrumentos útiles para la búsqueda de información, esquematización y análisis de información.

- El manejo de una segunda lengua, principalmente el inglés, también es importante, ya que mucha de la bibliografía para fundamentar las investigaciones se presenta en este idioma.

- Habilidades de abstracción, pensamiento sistemático, experimentación y colaboración.

Estas habilidades se ven reflejadas también en el Plan de Estudios del programa educativo de la Licenciatura en Ciencias de la Educación, que es el objeto de estudio de esta investigación, en donde el egresado tiene como meta poseer el dominio de los enfoques teóricometodológicos de investigación educativa para realizar proyectos de investigación y/o de intervención. (UAEH, 2014, Objetivos curriculares, párr. 8) que literalmente señala: "5.- Investigar e intervenir en la problemática educativa a partir de diferentes enfoques teóricosmetodológicos en proyectos de investigación para contribuir a la mejora educativa, en los ámbitos locales, regionales, nacionales e internacionales.

Todo esto nos permite cuestionar: ¿por qué si existen los espacios determinados de aprendizaje no se ve reflejado en las estadísticas de producción científica a través de tesis, artículos o congresos? Por ello se considera oportuno diagnosticar cuáles son las creencias y actitudes que tienen los estudiantes de la Licenciatura hacia su formación para el logro de dicha competencia. Lo cual podría dar pauta para que a futuro se pudieran diseñar intervenciones educativas pertinentes en estudios subsecuentes.

Con base en los planteamientos anteriores, la presente investigación se centra en el diseño de un instrumento para analizar las creencias y actitudes de un grupo de estudiantes de la Licenciatura en Ciencias de la Educación del Instituto de Ciencias Sociales y Humanidades de la Universidad Autónoma del Estado de Hidalgo hacia su formación para el logro de la competencia de investigación educativa, considerando estudiantes del programa educativo donde aplica el rediseño curricular 2014. 


\section{Marco Teórico}

La formación de investigadores es una tarea en la que se han centrado los países para promover políticas que se encaminen a la consolidación de la ciencia, como eje central para su avance y progreso (Miyahira, 2009; Sebastián, 2003).

De acuerdo con el Programa Sectorial de Educación (2013-2018), una de sus estrategias es garantizar que los planes y programas de estudio sean pertinentes y contribuyan a que los estudiantes puedan avanzar exitosamente en su trayectoria educativa, al tiempo que desarrollen aprendizajes significativos y competencias que les sirvan a lo largo de la vida, entre ellas, la de investigación (Secretaría de Educación Pública, 2013). La enseñanza de la investigación, como se señaló en la introducción, se promueve de forma tácita en los programas de educación superior, por lo que uno de los primeros espacios para su desarrollo es el aula y por ende, se convierte en una fuente de influencia clave para la formación de creencias y actitudes hacia ella.

También se debe considerar que las instituciones de educación superior son motores fundamentales para desarrollar la cultura y la ciencia de un país, a través de la formación de futuros investigadores, entre otros profesionales (Sánchez, 2002). Dicha formación está a cargo de los docentes, los cuales tienen un papel importante no solo en la enseñanza del nivel superior, sino desde la educación básica. Se busca en general, formar a ciudadanos que desarrollen competencias en la autonomía para seguir aprendiendo a lo largo de toda su vida (Mayor, 2009).

Para Rojas (2009), la labor docente y las condiciones concretas en que la ejercen tienen un alto grado de impacto en la manera en que los aprendices asumen, definen y participan de la investigación en las universidades. Es decir, una de las labores es promover en los alumnos actitudes positivas hacia la investigación. En este sentido, la actitud que los docentes puedan promover en sus alumnos tiene un papel importante para que se desarrollen ciertas habilidades hacia la investigación. Sánchez (2016) hace mención que las actitudes hacia el conocimiento y la investigación se describen como los intereses y motivaciones que los sujetos aprenden y desarrollan para conocer el mundo y acercarse a él con una mirada reflexiva y problematizadora. El desarrollo de las actitudes hacia el conocimiento y la investigación deben partir entonces de una metodología didáctica basada en enfoques constructivistas donde el aprendiz se ve motivado a aprender a partir de desarrollar actividades significativas y socialmente relevantes. Por ejemplo, a través del análisis de problemas reales, casos o el diseño de proyecto (Díaz Barriga y Hernández, 2010; Díaz Barriga, 2006; Sarabia, 1992). Centrar las actividades en la revisión y análisis de las investigaciones científicas formalmente publicadas es acercar las estrategias y productos desarrollados por expertos a estudiantes que son considerados novatos. Con estas acciones se está situando a los aprendices en una zona de desarrollo próximo y desde un sentido vigotskiano, la actitud positiva hacia el aprendizaje y desmitificación de las creencias falsas por unas científicas y formales se convierten en un instrumento psicológico que contribuye a un ambiente de aprendizaje adecuado y pertinente (Vygotsky, 1979).

Por lo anterior, se puede afirmar que estas habilidades investigativas no se pueden promover si el estudiante no se encuentra en un ambiente de aprendizaje que lo motive o estimule a aprender. Es decir, las creencias y actitudes pueden condicionar seriamente la situación didáctica, afectándola negativa o positivamente para el logro de los aprendizajes.

En este sentido, existen estrategias que se utilizan como medios para impulsar a dicha formación; en la siguiente lista se presentan algunas mediaciones planteadas por Aldana (2012), en el sentido de la formación en investigación.

- Experiencias de formación en los seminarios: En los seminarios suele generarse con intención formativa, pero también con carácter evaluativo, una serie de productos curriculares (ensayos, reportes de lectura, presentaciones orales, etc.) a los que el estudiante debe atender de manera simultánea al avance de su investigación. La cuestión es si esos productos curriculares resultan pertinentes en orden de apoyar la formación profesional, pero también de brindar herramientas para que los estudiantes se desempeñen con rigor científico, respecto a la investigación que tienen en proceso.

- Los coloquios como experiencia de formación: Los coloquios son eventos académicos, generalmente semestrales, en los que los estudiantes presentan sus avances de investigación y reciben comentarios de investigadores con experiencia, del propio tutor, y en algunos casos, de sus pares, compañeros estudiantes. Las principales intenciones formativas de los coloquios es primero que los estudiantes se familiaricen con la crítica como elemento fundamental para el enriquecimiento de los productos que se generan por vía de la investigación; en este caso, la que ellos mismos están realizando. Segundo, que a través de la exposición oral realicen una socialización de su conocimiento y en esa acción reconstruyan la interpretación de lo que saben, consolidando su aprendizaje.

- La relación de asesoría o dirección de tesis: La relación de asesoría como mediación fundamental en los procesos de formación para la investigación, la han descrito como la relación pedagógica ideal para llevar a cabo la función formadora donde un experto guía y lleva la dirección de un proyecto de investigación que es elaborado por el estudiante o tesista.

- Las acciones docentes: Las acciones docentes son múltiples y diversas; impactan en la calidad de los logros académicos, en la motivación para el trabajo (compromiso), en la imagen que los estudiantes construyen sobre lo que es el desempeño de un investigador, así como en la decisión de aspirar a ser (o no) uno de ellos. 
Por su parte, Villarreal y Guevara (1994) señalan que una estrategia posible de formación de investigadores, dentro de las universidades, es constituir núcleos de investigación integrados por investigadores en formación, ya sea alumnos de pregrado o posgrado bajo la dirección de un investigador formado; en este sentido, dentro de estos núcleos los alumnos tendrán la posibilidad de colaborar con el investigador de la institución en el proyecto y bajo la línea de investigación que éste esté desarrollando, lo que le permite irse formando con la dirección de un investigador experimentado, para que después el alumno pueda aplicar lo aprendido en la construcción de un proyecto propio.

Sin embargo, entre esta interacción dentro de los núcleos de investigación y la relación entre investigador experimentado y alumno en formación, se entiende que se debe crear un clima de confianza y no de superioridad por parte del investigador experimentado, sino más bien establecer una relación de ayuda con alumno, promoviendo entre otras cosas actitudes positivas hacia la investigación y bajo su asesoría, ir desarrollando ciertas habilidades para la investigación.

Con base en lo anterior se pueden definir algunos aspectos que consideramos relevantes para el fundamento del instrumento que se plantea diseñar como propósito de esta investigación. Si se van a valorar las actitudes y creencias de los estudiantes, es fundamental considerar a éstos en torno a la práctica educativa. Es decir, se toman como dimensiones esenciales, en primera instancia, el método de enseñanza y aprendizaje de esta competencia investigativa, así como la evaluación del aprendizaje, en segunda.

La tercera dimensión considerada es la articulación de las asignaturas centradas en las habilidades investigativas, así como su promoción transversal durante el desarrollo del currículo del programa. Señalando que el instrumento pretende abordar la actitud y creencias hacia la investigación en un contexto determinado. En este caso, dentro de espacios de formación universitaria. $Y$ en consecuencia considerar el programa educativo y la articulación de las asignaturas para la promoción de la competencia investigativa es esencial.

La cuarta dimensión, que se infiere, plantea rescatar lo que los autores señalados (Aldana, 2012; Villarreal y Guevara, 1994) abordan respecto a que el estudiante aprendiz pueda acercar con los expertos. Es decir, con el campo profesional o de acción donde se ponen en práctica formal las habilidades investigativas. Con base en estos planteamientos, a continuación, se describen con mayor detalle cada una de las dimensiones señaladas.

\section{Métodos de enseñanza y aprendizaje}

Dentro del acto didáctico, en cualquier contenido, incluyendo el de la formación de la competencia de investigación, se hacen presentes elementos de enseñanza y elementos de aprendizaje, los cuales regularmente son conocidos como métodos 0 estrategias. En este sentido, la Universidad Autónoma del Estado de Hidalgo (2014) plantea métodos innovadores acordes con enfoques de las teorías del aprendizaje constructivistas, los cuales demandan una actitud positiva y la desmitificación de creencias para contar con un ambiente de aprendizaje adecuado durante el proceso de construcción del conocimiento.

\section{Evaluación del aprendizaje}

Para esta investigación, nos sumamos a Morán (2003), quien define a la evaluación como el estudio del proceso de aprendizaje en un curso, un taller, un seminario, etc. con la finalidad de caracterizar los aspectos más destacados del mismo y, a su vez, los obstáculos que hay que enfrentar. El mismo autor, también considera a la evaluación como un medio para que los maestros y los alumnos cobren conciencia de la forma en que se aprendió (metacognición), lo cual conlleva al cuestionamiento de la herramienta para evaluar dicho proceso. Es por ello, que es fundamental recordar que la evaluación debe ser considerada como una transformación de la cultura escolar, donde es primordial pensar en el mejoramiento académico continuo, la reflexión y la autoevaluación, asimismo recordar que la evaluación debe servir específicamente en colaboración para una mayor productividad y la efectividad tanto de la enseñanza como del aprendizaje.

La UAEH (2014) destaca que la evaluación es parte fundamental para la calidad, por lo que la establece como una estrategia de mejora continua, no solo en procesos de evaluación interna, revisión, actualización, rediseño y creación de su oferta educativa sino en los procesos de enseñanza y aprendizaje que dan libertad a sus catedráticos para poder llevarla a cabo. Así, también la evaluación docente es otra de las acciones que son retomadas para encaminar sus esfuerzos hacia la calidad (UAEH, 2014). Por ello se considera un elemento esencial en torno a las actitudes y creencias hacia la investigación.

\section{Articulación entre las materias}

La articulación entre materias permite unificar contenidos y darle una secuencia a nivel macro, respecto a la formación de estudiantes de educación superior, para una mayor comprensión, profundidad y desarrollo lógico de la práctica de enseñanza, buscando garantizar la formación profesional paulatina, accediendo a contenidos progresivamente más complejos. Por ello, la universidad ha planteado materias institucionales que se dan en todas las carreras, una de ellas, centrada en habilidades investigativas. Así mismo, cada programa de estudios incluye materias y contenidos en el mismo sentido temático o relacionado con él, de tal forma que competencias como la de investigación se promueven de forma transversal a lo largo de todo el programa (UAEH, 2014). En consecuencia, si se promueve directa o indirectamente en los temas de los programas de la formación universitaria se podría conjeturar respecto a 
que esto debería promover una actitud positiva hacia la investigación.

\section{Campo profesional del egresado}

El campo profesional está compuesto por escenarios en los que el profesional es capaz de posicionarse a través de las habilidades, conocimientos y competencias que ya posee (UAEH, 2014). Se asume, desde los perfiles de egreso, que el recién profesional será capaz de desempeñarse en organismos, entidades y establecimientos educativos formales y no formales, oficiales y no oficiales y en todo tipo de organización o entidad que realice actividades vinculadas con la educación, ya sea en forma presencial o a distancia, entre ellas las habilidades investigativas. Es decir, estar consciente que, aunque no se pretenda ser investigador, las habilidades de investigación son necesarias y clave para el ejercicio de la profesión. Por ello, es necesario indagar al respecto en torno a las actitudes hacia la investigación en este contexto.

\section{Cuerpo del Reporte}

Por sus pretensiones es un estudio descriptivo enfocado en el sustento y descripción del diseño de un instrumento cuantitativo a través de una escala Likert, donde se inició la puntuación de las opciones de respuesta en cero (0) para evitar la tendencia a elegir la opción tres (3) como punto neutro. Entre más se acerque la puntuación a cero las actitudes y creencias serán negativas.

El cuestionario, como se mencionó, consideró las siguientes dimensiones, a partir de las cuales se diseñaron los indicadores e ítems. La primera versión del instrumento está compuesta por 56 ítems; 28 para creencias y 28 para actitudes, divididas en dimensiones de métodos de enseñanza y aprendizaje, articulación con las materias, evaluación del aprendizaje, y vínculo con el campo profesional.

Los participantes fueron un total de 73 personas encuestadas, 11 hombres y 62 mujeres, con edades entre los 20 y 23 años, se consideran de clase media, ya que tienen la posibilidad de estudiar. Alrededor de $40 \%$ son foráneos, provenientes de poblados circundantes a Pachuca y del estado de México.

En este sentido, la muestra es no probabilística, porque su elección parte de la relación entre características que se relacionan con la investigación, debido a que los alumnos de la generación en la que se está analizando pertenecen al rediseño del programa.

Para valorar la fiabilidad del instrumento se realizó un análisis Alfa de Cronbach, que permite estimar la fiabilidad de un instrumento de medida a través de un conjunto de ítems que se espera que midan el mismo constructo o dimensión teórica. El resultado fue favorable, aunque no estadísticamente significativo, con un valor de .771, que según el análisis Cronbach entre más se acerque a 1 mayor es su fiabilidad.

Se valoran también, aspectos cualitativos a través de observaciones o comentarios puntuales de alumnos y docentes, que permitan generar un ejercicio de comprensión de la realidad educativa en torno a las creencias y actitudes hacia la investigación en el contexto educativo objeto de estudio, lo cual también permitió mejorar el instrumento durante su rediseño.

\section{Discusión y Resultados}

Las iniciativas sobre la promoción de la investigación son fundamentales y necesarias para fortalecer las instituciones educativas de México, quien desafortunadamente se encuentra en un nivel preocupante al respecto. La investigación puede considerarse uno de los motores necesarios para el desarrollo de una sociedad y en ese sentido, México debe generar iniciativas que consoliden la promoción de esta competencia en los espacios educativos.

Para esta investigación en particular resultó indispensable indagar sobre las creencias y actitudes hacia la investigación educativa que existen en el contexto institucional de una universidad pública, de manera puntual en la Licenciatura en Ciencias de la Educación. Lo cual ofrece los elementos necesarios para diseñar pautas de intervención educativa que aseguren y consoliden la competencia de investigación en los estudiantes y docentes de la misma.

Para ello se presenta el rediseño del cuestionario inicial, con base en el análisis de resultados de la primera versión. En resumen, para la presentación de los resultados de esta primera aplicación los puntajes brutos se convirtieron en valores Z:

\section{Gráfica 1}

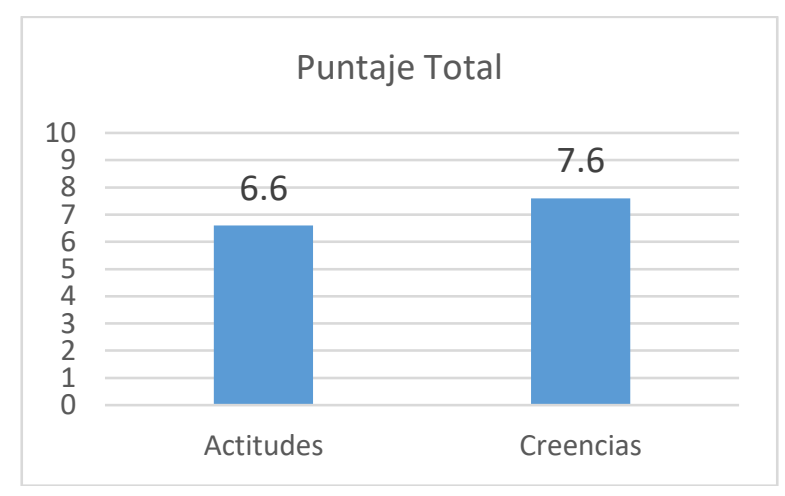

La gráfica 1 muestra los puntajes referentes a las creencias y actitudes de la competencia en investigación educativa, en los cuales se puede reflejar de manera general que los alumnos no tienen actitudes muy positivas hacia la investigación, ya que el valor se encuentra en un promedio de 6.6, que se considera un valor bajo, al igual que las creencias que están solo por un punto arriba de las actitudes. Es decir, no hay un ambiente óptimo o adecuado desde una visión socio constructivista del aprendizaje. 


\section{Gráfica 2}

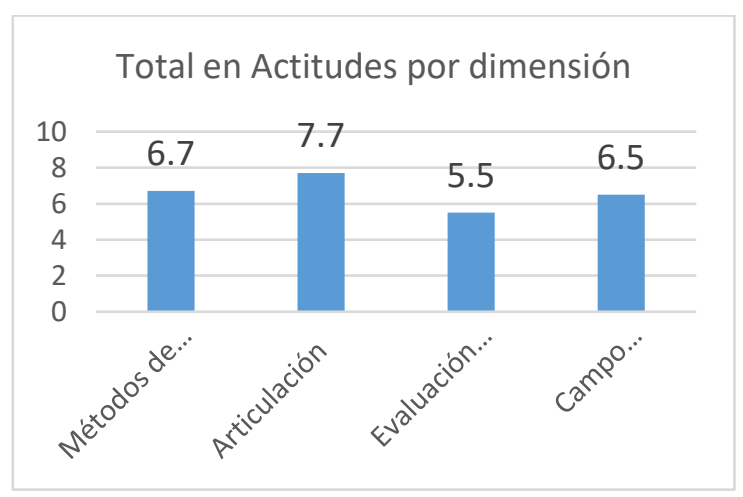

La gráfica 2 muestra cómo se visualizan las actitudes que tienen los alumnos respecto a las 4 dimensiones que se buscaban analizar, donde el nivel más bajo se presenta en la dimensión de evaluación, es decir se puede conjeturar en torno a que los estudiantes consideran que al evaluar su aprendizaje las materias de investigación no se toman en cuenta sus intereses y características, o quizás los criterios se consideran ambiguos por lo que buscan evitar presentar proyectos de investigación.

\section{Gráfica 3}

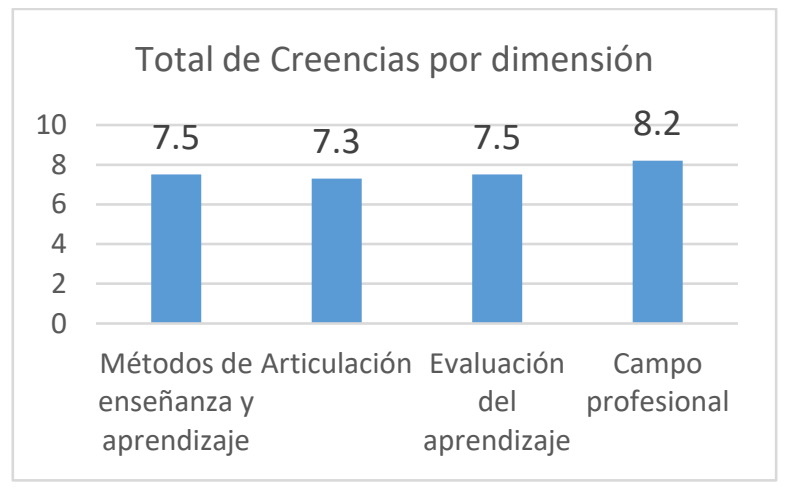

La gráfica anterior muestra cómo los alumnos tienen creencias que se pueden considerar regulares en el campo profesional, reconociendo que adquirir y desarrollar la competencia en investigación educativa, contribuye para la toma de decisiones en cualquier sector educativo. De ahí la importancia de ésta, ya que consideran que la investigación educativa permite que el Licenciado en Ciencias de la Educación tenga mejores y más oportunidades de trabajo; además de que permite la resolución de problemas educativos y sociales tales como la retención de la población que emigra en busca de mejores condiciones de vida, entre otros.

Cabe señalar que los resultados permiten establecer la importancia de consolidar el diseño del instrumento a través de una validación con una muestra más amplia que permita obtener un mejor puntaje en la fiabilidad y obtener resultados estadísticamente significativos ante una muestra que represente una población más amplia dentro del mismo nivel educativo. Con la intención de contar con datos que permitan generar propuestas de intervención que busquen promover una actitud positiva hacia la investigación, así como la desmitificación de falsas creencias en torno a ella.

Finalmente, se hace una invitación para contribuir en los datos recogidos en diversos contextos, con la intención de realizar una validación más amplia y adecuada del instrumento en contextos similares universitarios para realizar estudios comparados.

Es importante señalar que también se trabajará en la versión para docentes, la cual ayudará a completar de forma más adecuada el ejercicio de acercamiento al fenómeno de las actitudes hacia la investigación.

\section{Anexo}

Cuestionario rediseñado

Creencias y actitudes hacia la investigación en estudiantes de la Licenciatura en Ciencias de la Educación

Instrucciones: A continuación, encontrarás preguntas referentes a las creencias y actitudes hacia las tareas investigativas, por lo que no se consideran respuestas correctas o incorrectas.

Toda información es anónima y se reserva para uso exclusivo de la investigación, por lo que es importante mencionar que todo lo que escribas es totalmente confidencial, por favor contesta con toda la sinceridad posible. De ante mano gracias por tu importante participación.

Edad: Sexo:

Semestre: Grupo:

Señala el puntaje del 1 al 10 que consideras más adecuado para cada afirmación. A mayor puntaje más adecuado. No hay respuestas correctas o incorrectas.

Práctica docente orientada a la investigación La mayoría de los docentes...

1. Promueve actividades relacionadas con la investigación, aun cuando no sean materias propias de esta área

2. Nos permite generar propuestas de investigación relacionadas con nuestros intereses

3. Utiliza materiales didácticos para promover el interés sobre actividades de investigación.

4. Emplea métodos y técnicas orientados al desarrollo de las competencias en investigación educativa.

5. Promueve la revisión de reportes de investigación, por ejemplo: artículos, tesis y ponencias

6. Facilitan la construcción de competencias investigativas en los estudiantes

7. Que evalúan los proyectos de investigación lo hacen con criterios claros y específicos (rúbricas, listas de cotejo)

Valoración curricular

Los contenidos de las asignaturas de investigación ....

8. Son actuales 
9. Son pertinentes

10. Son socialmente relevantes

11. Son aburridos

12. Son complicados

13. Contribuyen al desarrollo de la misión, objetivos curriculares y perfil de egreso

14. Son importantes para las demás asignaturas

Actitud hacia la investigación

15. Hacer investigación es sencillo

16. La investigación es una herramienta de apoyo para el aprendizaje

17. Me gusta el trabajo sistemático de la investigación

18. Me gusta buscar información sobre algún tema de investigación

19. Me interesa conocer los enfoques teóricos que intervienen para realizar un proyecto de investigación

20. Me interesa conocer sobre los diferentes enfoques metodológicos para realizar un proyecto de investigación

21. Evito presentar proyectos de investigación para que me evalúen

22. Me interesa conocer los campos de conocimiento de la investigación educativa

23. Me interesa trabajar de manera cooperativa y colaborativa en proyectos de investigación

24. Me interesa exponer en congresos educativos los resultados de investigaciones en las que he colaborado

25. Si tuviera la oportunidad de participar en alguna investigación, me gustaría publicar artículos sobre los hallazgos

Competencia Investigativa

26. Identifico los modelos cualitativos y cuantitativos para generar proyectos de investigación pertinentes

27. Puedo utilizar diferentes procesadores de textos, software, paquetes estadísticos como recursos para el desarrollo de la investigación

28. Estoy capacitado(a) para generar proyectos de investigación e intervención en problemáticas de mi disciplina a partir de diferentes enfoques teóricometodológicos

29. Puedo desarrollar propuestas de investigación para solucionar un problema en específico o contribuir a la generación de conocimiento

30. Considero que tengo conocimientos metodológicos sólidos para hacer investigación

Selecciona la opción que te resulte adecuada o en el caso de las preguntas abiertas contesta lo que se te solicita.

31. Pensar en involucrarme en actividades de investigación me produce... Nota: Puedes seleccionar más de una opción.
a) Interés
b) Agrado
c) Entusiasmo
d) Pereza

\section{e) Desagrado \\ f) Ansiedad}

32. ¿Cuál de las siguientes opciones elegirás para titularte?

a) Examen general de conocimientos

b) Tesis (tendría que ir al último)

c) Otra: (especifique)

33. ¿Cuál es la razón por la que elegirás esa opción de titulación?

34. ¿Para qué consideras importante desarrollar competencias investigativas?

\section{References}

Aldana de Becerra, Gloria Marlen (2012) "La formación investigativa: su pertinencia en pregrado" en Revista Virtual Universidad Católica del Norte. Vol. 35, pp. 367-379. Colombia: Católica del Norte

Díaz-Barriga Arceo, Frida. y Hernández Rojas, Gerardo (2010) Estrategias Docentes para un aprendizaje significativo: Una interpretación constructivista. México, Mc Graw Hill

Díaz-Barriga Arceo, Frida (2006). Enseñanza situada: vínculo entre la escuela y la vida. México: McGrawHill.

Estrada Molina, Odiel (2014) "Sistematización teórica sobre la competencia investigativa" en Revista Electrónica Educare, Vol. 18, núm. 2, pp. 177-194. Costa Rica: Universidad Nacional Heredia

Mayor Ruiz, Cristina (2009) "Nuevos retos para una universidad en proceso de cambio ¿Puedes ser los profesores (principiantes) los protagonistas?" en Revista de curriculum y formación del Profesorado, Vol. 13, núm. 1 pp. 61-77. Granada: Universidad de Granda

Miyahira Arakaki, Juan Manuel (2009) "La investigación formativa y la formación para investigación en el pregrado" en Revista Médica Herediana. Vol. 20, núm. 3, pp.119-122. Perú: Universidad Peruana Cayetano Heredia

Morán Oviedo, Porfirio (Comp.) (2003) Docencia e Investigación en el Aula. Una relación imprescindible. México: Tercera época, Pensamiento Universitario, CESU, UNAM.

Moreno Bayardo, María Guadalupe (2011) "La formación de investigadores como elemento para la consolidación de la investigación en la universidad" en Revista de la Educación Superior. Vol. XL 2, núm. 158, pp. 59-78, México: Asociación Nacional de Universidades e Instituciones de Educación Superior

Rojas Betancur, Héctor Mauricio (2009) "Formar investigadores e investigadoras en la universidad: optimismo e indiferencia juvenil en temas científicos" en Revista Latinoamericana de Ciencias Sociales, Niñez y Juventud, Vol. 7 núm. 2, Suppl.1, pp. 1595-1618. Manizales: Universidad de Manizales

Sarabia Heydrich, Bernabé. (1992) "El aprendizaje y la enseñanza de actitudes" en Cesar Coll, Juan Ignacio Pozo, B. Sarabia y E. Valls. Enseñanza y aprendizaje de conceptos, procedimientos y actitudes. pp.133-197, Madrid: Santillana,.

Sánchez-Castañeda, Alfredo (2002) "Los Retos de la Educación Superior: Hacia una política de Estado" [En línea] México, disponible en: https://archivos.juridicas.unam.mx/www/bjv/libros/1/341/19.pdf $\quad[20$ de enero de 2018] 
Sánchez Pérez, Samuel Haroldo (2016) Actitud hacia la investigación en alumnos y docentes universitarios de Tuxtla Gutiérrez Chiapas. Tesis de Maestría. México: Universidad de Montemorelos.

Secretaría de Educación Pública (2013) "Programa Sectorial de Educación 2013-2018" [En línea] México, disponible en: http://www.sep.gob.mx/work/models/sep1/Resource/4479/4/images/P ROGRAMA_SECTORIAL_DE_EDUCACION_2013_2018_WEB.pd f [23 de enero de 2018]

Sebastián, Jesús (2003) "Estrategias de cooperación universitaria para la formación de investigadores en Iberoamérica" en Organización de Estados Iberoamericanos para la Educación la Ciencia y la Cultura [En línea] Disponible en: https://www.oei.es/historico/superior/jsebastian.pdf [23 de enero de 2018]

Universidad Autónoma del Estado de Hidalgo (2014) "Plan de Estudios Licenciatura en Ciencias de la Educación"[En línea] México, disponible https://www.uaeh.edu.mx/campus/icshu/oferta/licenciaturas/lic_educac ion_plan2014.html [23 de enero de 2018]

Universidad Autónoma del Estado de Hidalgo. (2013) "Reglamento Escolar de la Universidad Autónoma del Estado de Hidalgo" [En línea] México, disponible en: https://www.uaeh.edu.mx/adminyserv/dir_generales/juridica/reglament o_escolar09.htm [23 de enero de 2018]

Villarreal, Diana y Guevara C., José Luis (1994) "Una Experiencia en Formación de Investigadores. Núcleos de Investigadores en la Universidad Autónoma de Tamaulipas" en Revista de la Educación Superior. Vol. XXIII, núm. 92, pp. 7-12, México: Asociación Nacional de Universidades e Instituciones de Educación Superior

Vygotsky Semiónovich, Lev (1979) El desarrollo de los procesos psicológicos superiores. Barcelona: Crítica. 